\title{
Disección coronaria espontánea. Primer reporte a nivel nacional
}

\author{
Dres. Rafael Mila, Juan Albistur, Juan Pablo Bachini, \\ Álvaro Niggemeyer, Ariel Durán
}

\begin{abstract}
Resumen
La disección coronaria espontánea (DCE) es una causa infrecuente de síndrome coronario agudo (SCA), de presentación principalmente en mujeres de mediana edad. Se realizó una revisión de la base de datos institucional, desde la incorporación en nuestro centro de tecnología para adquisición de imagen intracoronaria hasta la actualidad (julio de 2013 a diciembre de 2019). Se encontró DCE en 13 de 4.706 coronariografías diagnósticas (0,3\%). Presentamos angiografía y ultrasonografía intracoronaria (IVUS) representativa de lo observado en la serie. La edad media de presentación fue $52 \pm$ 14 años, 11 de los 13 casos eran mujeres. El tiempo medio de seguimiento fue $39 \pm 27$ meses. La forma clínica de presentación más frecuente fue SCA sin elevación del ST (6/13). El vaso más frecuentemente afectado fue la arteria descendente anterior $(n=8)$. En todos los casos se registró elevación significativa de troponinas ( $>$ percentil 99). Se realizó tratamiento conservador en 11 casos y angioplastia exitosa en dos. Un paciente que recibió tratamiento conservador falleció durante la internación. Se requirió de imagenología intracoronaria en nueve de los 13 casos. Se presenta el primer reporte nacional de DCE, describiendo sus características clínicas, imagenológicas, tratamiento y evolución.

Palabras clave: $\quad$ DISECCIÓN CORONARIA ESPONTÁNEA

SÍNDROME CORONARIO AGUDO

ULTRASONOGRAFÍA INTERVENCIONAL
\end{abstract}

\section{Spontaneous coronary dissection. First nationwide report}

Summary

Spontaneous coronary dissection is an infrequent cause of acute coronary syndrome, particularly in middle-aged women. We made review of the database of our center since the date of acquisition of intracoronary imaging techniques in our laboratory (July 2013 - December 2019). Coronary spontaneous dissection was found in 13 patients of 4706 diagnostic coronary angiograms $(0.3 \%)$. We present angiography and coronary ultrasonography studies representative of this case series. The mean age of presentation was $52 \pm 14$ years, 11 of 13 of the cases were women. The mean follow-up time was $39 \pm 27$ months. The most frequent clinical presentation was acute coronary syndrome without ST elevation 6/13. The most frequently affected vessel was the anterior descending artery $(n=8)$. In all cases, troponins above the 99 percent were found. Conservative treatment was performed in 11 cases and successful angioplasty in the other two cases. One patient under conservative treatment died during hospitalization. The use of intracoronary imaging was required in 9 of the 13 cases. In conclusion, we present for the first time, a national registry of spontaneous coronary dissection describing its clinical, image, treatment and evolution characteristics.

Key words: $\quad$ SPONTANEOUS CORONARY DISSECTION

ACUTE CORONARY SYNDROME

INTERVENTIONAL ULTRASONOGRAPHY

Servicio de Hemodinamia, Centro Cardiovascular Universitario, Hospital de Clínicas. Montevideo, Uruguay.

Correspondencia: Dr. Rafael Mila. Avda. Italia S/N. Hospital de Clínicas. Montevideo, Uruguay.

Correo electrónico: r1000a@gmail.com

Los autores declaran no tener conflictos de intereses.

El presente estudio no tiene fuentes de financiamiento. 


\section{Dissecção coronariana espontânea. Primeiros relatórios em todo o país}

\section{Resumo}

A dissecção coronariana espontânea é uma causa pouco frequente da síndrome coronariana aguda, principalmente em mulheres de meia idade. Realizamos uma revisão do banco de dados de nosso centro a partir da data de aquisição das técnicas de imagem intracoronária em nosso laboratório (julho de 2013 a dezembro de 2019). Dissecção coronariana espontânea foi encontrada em 13 pacientes de 4706 angiografias coronárias diagnósticas $(0,3 \%)$. Apresentamos angiografia coronariana representativa e estudos ultrassonográficos desta série de casos. A idade média de apresentação foi de $52 \pm 14$ anos, 11 dos 13 casos eram do sexo feminino. O tempo médio de acompanhamento foi de $39 \pm 27$ meses. A apresentação clínica mais frequente foi síndrome coronariana aguda sem supradesnivelamento do segmento ST 6/13. O vaso mais acometido foi a artéria descendente anterior $(n=8)$. Em todos os casos, as troponinas foram encontradas acima de $99 \%$. O tratamento conservador foi realizado em 11 casos e a angioplastia em 2, com sucesso. Um paciente em tratamento conservador morreu durante a internação. O uso de imagens intracoronárias foi necessário em 9 dos 13 casos. Concluindo, relatamos, pela primeira vez, um registro nacional de dissecção coronariana espontânea de que descreve suas características clínicas, de imagem, de tratamento e de evolução.

Palavras chave: $\quad$ DISSECÇÃO CORONARIANA ESPONTÂNEA

SÍNDROME CORONARIANA AGUDA

ULTRASSONOGRAFIA DE INTERVENÇÃO

\section{Introducción}

La disección coronaria espontánea (DCE) es una causa importante de síndrome coronario agudo (SCA), especialmente en mujeres de joven y mediana edad. Es nueve veces más frecuente en mujeres debido a sus causas predisponentes, como ser la displasia fibromuscular, embarazo y terapia hormonal (estrógeno y progesterona) ${ }^{(1)}$. La verdadera prevalencia de esta enfermedad es desconocida debido al subdiagnóstico, pero ha sido reportada desde $0,1 \%$ a $4 \%$ de los pacientes que presentan un $\mathrm{SCA}^{(1)}$. Sin embargo, en estudios contemporáneos se ha visto una incidencia de $24 \%$ a $35 \%$ en mujeres menores de 60 años ${ }^{(2)}$.

La DCE se define como la separación de las capas de la pared arterial coronaria, secundaria a una hemorragia intramural no traumática. La apariencia angiográfica difiere de acuerdo al mecanismo fisiopatológico. A este respecto existen dos teorías: a) inside-out, que lleva a la imagen clásica de múltiples lúmenes radiolúcidos y tinción de la pared arterial con contraste o lavado lento, que crea una falsa luz que puede estar acompañada o no de un desgarro intimal. Se puede originar a partir de una hemorragia por rotura de vasa vasorum intramural, o bien a partir de un desgarro intimal. La separación de capas puede ocurrir entre la íntima y la media o entre la media y la adventicia ${ }^{(3)}$; b) outside-in, donde se observa una estrechez luminal debida a compresión extrínseca, que de ser focal, puede ser interpretada como de causa aterosclerótica. Esta es en realidad la forma más frecuente y se denomina hematoma intramural. Se observa un estrechamiento local o difuso del vaso, sin doble luz. Cuando esto ocurre, típi-
Principales aportes

Es la primera vez que se reporta a nivel nacional la incidencia de la disección coronaria espontánea. Se trata de un importante diagnóstico diferencial del síndrome coronario agudo principalmente en mujeres de mediana edad. Es una entidad subdiagnosticada en todo el mundo, por lo que es necesario incrementar el índice de sospecha. La utilización de imagenología intracoronaria (IVUS u OCT) es imprescindible para el diagnóstico en muchos casos. Se muestran los patrones angiográficos e imágenes de IVUS característicos de la serie de casos reportada.

camente el vaso incrementa su tortuosidad y se generan ángulos rectos en su trayecto que le dan el aspecto de imagen "en sacacorcho" o "tirabuzón". Otras formas que llevan al subdiagnóstico son la existencia de estrechez leve, así como el involucramiento de ramas distales y pequeñas $\operatorname{arterias~}^{(4)}$. La fisiopatología no se encuentra completamente aclarada. Aparentemente existe una arteriopatía de base que debilita la pared arterial en la mayor parte de los casos.

La incidencia de esta enfermedad es desconocida en nuestro país, principalmente por la falta de disponibilidad de imagenología intracoronaria en todos los centros, ya que actualmente no es una prestación obligatoria según el Fondo Nacional de Recursos, si bien está prevista su inclusión. En este trabajo reportamos las DCE, su incidencia, clasificación, características clínicas, angiográficas e imagenológicas, tratamiento y evolución, a partir de su 
detección mediante angiografía o ultrasonografía intracoronaria (IVUS), o ambas, cuando se consideró necesario para el diagnóstico definitivo.

\section{Material y método}

Se realizó un estudio descriptivo y retroprospectivo, mediante una revisión de la base de datos del Centro Cardiovascular Universitario, que incluyó el diagnóstico de DCE, hematoma mural y hematoma intramural. Se excluyeron explícitamente las disecciones iatrogénicas mediante revisión de las imágenes de angiografía. En caso de existir duda, se realizó una revisión por dos hemodinamistas de forma independiente, y en caso de discrepancia, la consulta con un tercero, el de mayor experiencia en el centro. Se incluyó el período julio de 2013 a diciembre de 2019. Se revisaron los registros clínicos, paraclínicos, angiografía e imagenología intracoronaria de los casos seleccionados. Se realizó seguimiento telefónico y revisión de la historia clínica de los pacientes para obtener datos de la evolución. Dependiendo de los hallazgos angiográficos, se clasificaron cuatro tipos diferentes de $\mathrm{DCE}^{(1,5)}$ :

- Tipo 1: típico "flap" radiolúcido con imagen de doble luz debido a un defecto de relleno lineal, frecuentemente asociado a retención de contraste.

- Tipo 2: estenosis larga y suave localizada principalmente en el sector medio o distal de la arteria que se subdivide en dos tipos:

- Tipo 2a: el vaso distal mantiene un calibre normal.

- Tipo 2b: la estenosis alcanza el final del vaso, respetando más o menos el aspecto anatómico del vaso.

- Tipo 3: angiográficamente indistinguible de la aterosclerosis focal o tubular; se requiere IVUS o tomografía de coherencia óptica (OCT) para demostrar la presencia de un hematoma intramural o doble luz, o ambos, o repetición de la angiografía para documentar la desaparición.

- Tipo 4: oclusión total del vaso (habitualmente distal), cuando se han excluido causas de embolia. Para definir este tipo es necesario documentar la curación del vaso.

Los datos son presentados como frecuencias absolutas, relativas, media $\pm \mathrm{DE}$ (desvío estándar).

\section{Resultados}

Se encontró DCE en 13 pacientes de 4.706 coronariografías diagnósticas realizadas en nuestro centro en un período de 6,4 años, lo cual representa $0,3 \%$
Tabla 1. Caracterización clínica y forma de presentación.

\begin{tabular}{lc}
\hline N & 13 \\
Sexo femenino & 11 \\
Edad (años) & $52 \pm 14$ \\
Seguimiento (meses) & $29 \pm 27$ \\
Fallecidos & 1 \\
HTA & 5 \\
Tabaquismo & 10 \\
Diabetes mellitus & 1 \\
Dislipemia & 4 \\
Predisponente & 4 \\
SCASEST & 6 \\
SCACEST transitoria & 2 \\
SCACEST & 4 \\
MS & 1 \\
\hline $\begin{array}{l}\text { HTA: hipertensión arterial; SCASEST: síndrome coronario } \\
\text { agudo sin elevación del ST; SCACEST transitoria: síndrome } \\
\text { coronario agudo con elevación del ST transitoria; SCACEST: } \\
\text { síndrome coronario agudo con elevación persistente del ST; } \\
\text { MS: muerte súbita. }\end{array}$ \\
\hline
\end{tabular}

de las coronariografías diagnósticas. Si se toma exclusivamente en cuenta a los pacientes con SCA, este porcentaje asciende a $0,6 \%$.

La edad media de presentación fue $52 \pm 14$ años, 11 de los 13 casos eran mujeres. El tiempo medio de seguimiento fue $39 \pm 27$ meses. Durante el seguimiento se registró el fallecimiento de una paciente internada, de 83 años. Los restantes pacientes permanecen vivos y asintomáticos hasta la fecha. En la tabla 1 se resumen las características clínicas de la serie. Se destaca que se identificó factor posible predisponente o desencadenante en cuatro pacientes: virus de la inmunodeficiencia humana (VIH), pancreatitis aguda en curso, desprendimiento prematuro de placenta normoinserta (DPPNI) con óbito fetal y embarazo ectópico complicado con sangrado que requirió cirugía de urgencia.

La forma clínica de presentación más frecuente fue el SCA sin elevación del ST (6/13) (figura 1). La frecuencia del tipo de disección se muestra en la figura 2. Destacamos que no se registraron disecciones tipo 4. En nueve casos hubo afección de vaso único y afección de más de un vaso en cuatro casos. $\mathrm{El}$ vaso más frecuentemente afectado fue la arteria descendente anterior $(n=8)$. En todos los casos se registró elevación significativa de troponinas $>$ percentil 99. Al momento de la angiografía hubo flujo 


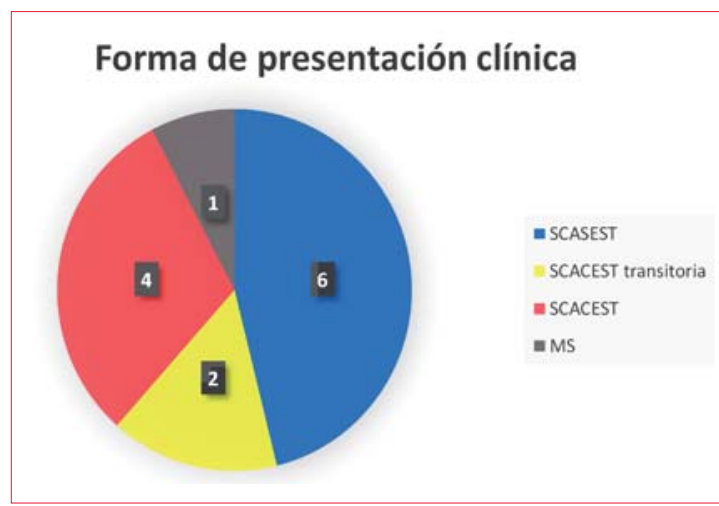

Figura 1. SCASEST: síndrome coronario agudo sin elevación del ST; SCACEST transitoria: síndrome coronario agudo con elevación transitoria del segmento ST; SCACEST: síndrome coronario agudo con elevación permanente del segmento ST; MS: muerte súbita.

Tabla 2. Características angiográficas y clasificación del tipo de disección coronaria espontánea.

\begin{tabular}{lc}
\hline Afectación arterial & Frecuencia \\
\hline Multivaso & 4 \\
TCI & 4 \\
ADA & 8 \\
ADi & 1 \\
ACX & 1 \\
AMO & 2 \\
ACD & 2 \\
\hline Flujo TIMI & \\
\hline 0 & 0 \\
1 & 0 \\
2 & 1 \\
3 & 12 \\
\hline Clasificación DCE & \\
\hline 1 & 4 \\
$2 \mathrm{a}$ & 2 \\
$2 \mathrm{~b}$ & 2 \\
3 & 5 \\
\hline & \\
\hline & \\
\hline & \\
\hline & \\
\hline & \\
\hline
\end{tabular}

TCI: tronco de coronaria izquierda; ADA: arteria descendente anterior; ADi: arteria diagonal; ACX: arteria circunfleja; AMO: arteria marginal obtusa; ACD: arteria coronaria derecha; DCE: disección coronaria espontánea.

TIMI 3 en todos los casos, excepto uno que fue TIMI 2. Respecto al tratamiento, fue conservador en 11 casos y en dos casos se realizó angioplastia (en un caso por la presentación como muerte súbita y en el otro por isquemia persistente en contexto de SCA

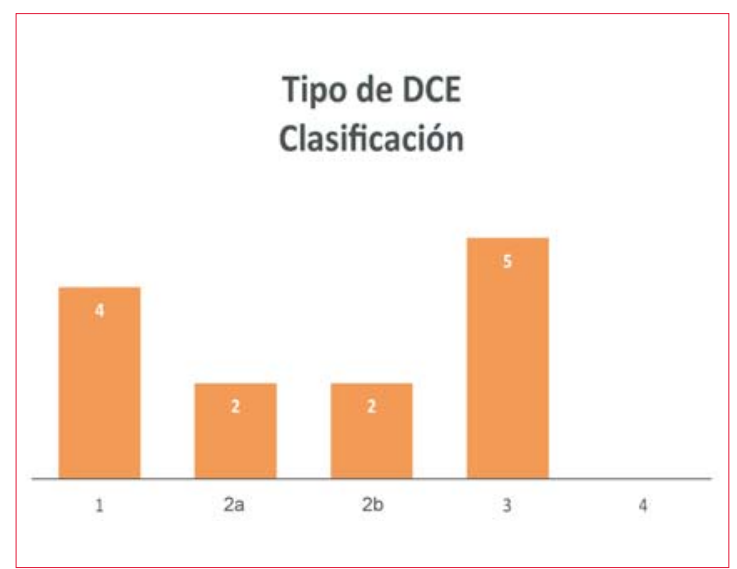

Figura2. Frecuencia de presentación angiográfica de los casos de disección coronaria espontánea de la serie, clasificados en tipo 1, 2a, 2b, 3 y 4 .

con elevación del ST). Ambas angioplastias fueron exitosas, sin complicaciones. Se requirió la utilización de imagenología intracoronaria en nueve de los 13 casos y fue IVUS. El tratamiento conservador consistió en realizar doble antiagregación plaquetaria con ácido acetil-salicílico y clopidogrel a dosis habituales. En todos los casos se utilizó como criterio de tratamiento alcanzar objetivos de prevención secundaria de enfermedad aterosclerótica. A modo ilustrativo se presenta la angiografía e imagenología intracoronaria de los casos clínicos más representativos (figura 3).

\section{Discusión}

Este es el primer reporte a nivel nacional que analiza la incidencia de DCE. La frecuencia encontrada se encuentra dentro los márgenes esperados ${ }^{(1)}$. La utilización de la imagenología intracoronaria ha permitido diferenciar los diferentes patrones angiográficos, permitiendo elevar el índice de sospecha. Si bien el IVUS tiene menor resolución espacial que la OCT (con menor sensibilidad para detectar disrupciones pequeñas de la íntima arterial), presenta mayor penetrancia en el tejido (4 a $8 \mathrm{~mm}$ ), lo que permite visualizar completamente la pared del vaso y evaluar la extensión del hematoma intramural ${ }^{(4)}$. Se requirió utilizar imagenología intracoronaria en nueve casos, siendo determinante en el diagnóstico. En los cuatro casos restantes, la presentación clínica y la angiografía fueron suficientes. A pesar de que la realización de imagen intracoronaria puede llevar a un incremento en el riesgo de disección iatrogénica, IVUS y OCT se han utilizado con seguridad bajo la recomendación de ser extremadamente cuidadoso en cualquier intervención con catéter en este escenario, realizando esta técnica solamente cuando existe indicación ${ }^{(6,7)}$. En este trabajo no se 


\begin{tabular}{lc} 
Tabla 3. Tratamiento y evolución. & \\
\hline Tratamiento & Frecuencia \\
\hline Conservador & 11 \\
Angioplastia & 2 \\
CRM & 0 \\
\hline Evolución & \\
\hline Uso de IVUS & 9 \\
Angiografía de control & 3 \\
Muerte & 1 \\
Recurrencia & 0 \\
\hline $\begin{array}{l}\text { CRM: cirugía de revascularización miocárdica; IVUS: ultraso- } \\
\text { nografía intracoronaria. }\end{array}$
\end{tabular}

observaron complicaciones por la utilización de IVUS.

La edad media de presentación fue similar a la del registro multicéntrico prospectivo más importante llevado adelante hasta el momento con 750 ca$\operatorname{sos}^{(8)}$. De forma concordante, encontramos una clara predominancia del sexo femenino, en quienes es más frecuente la DCE como causa no aterosclerótica de $\mathrm{SCA}^{(9)}$. Si bien en el pasado se otorgaba al embarazo un rol etiopatogénico central, las series modernas han ampliado la identificación de predisponentes y desencadenantes, por lo que ha descendido su importancia relativa. Actualmente se considera que contribuyen a la patogenia una combinación de factores genéticos, hormonales, arteriopatía subyacente o enfermedad inflamatoria sistémica, en que el estrés físico o mental juega un rol importante como desencadenante, siendo reconocido hasta en $80 \%$ de los $\operatorname{casos}^{(10)}$. En esta serie, encontramos cuatro casos con enfermedad predisponente, dos de ellos vinculados al embarazo (DPPNI y embarazo ectópico) y dos vinculados a inflamación: pancreatitis aguda (aguda) y VIH (crónica). No se realizó la pesquisa de fibrodisplasia muscular. Si bien no hay consenso en que esta enfermedad se deba investigar de manera sistemática, es un hallazgo muy frecuente ${ }^{(10)}$.

En la era previa a la imagenología intracoronaria, se consideraba que el hallazgo angiográfico más frecuente era encontrar a nivel del vaso culpable una o múltiples luces con retención de contraste, lo que hoy se clasifica como disección tipo 1. Este tipo de disección se encontró en cuatro pacientes. En las series más modernas se considera que su prevalencia no supera el $20 \%$. Si bien se trata de números pequeños, la sobrerrepresentación de este tipo de disección sugiere el subdiagnóstico de los tipos más frecuentes, como son las de tipo 2. El hecho de no haber observado disecciones tipo 4, también sugiere subdiagnóstico.

Se encontró elevación de troponinas en todos los casos. La elevación de biomarcadores es un hecho casi constante, existen reportes de series que muestran elevación en $100 \%$ de los casos ${ }^{(11)}$.

La evidencia respecto al tratamiento de estos pacientes no es tan abundante como para la coronariopatía aterosclerótica. En los inicios, se tendía a tratar la DCE bajo los mismos principios que la complicación de placa aterosclerótica, es decir, realizando revascularización percutánea. Hoy en día se considera que el tratamiento debe ser diferente. Se optó por un tratamiento conservador en 11 de los 13 casos, conducta acorde con las recomendaciones actuales. Se ha demostrado que quienes muestran estabilidad clínica al momento del diagnóstico, tienen buenos resultados con un manejo conservador y evolucionan a la curación espontánea. En la serie de Vancouver, se encontró que los 79 pacientes con seguimiento angiográfico mostraron curación completa en un promedio de 161 días $^{(12)}$. A diferencia de lo que sucede en los SCA de causa aterosclerótica, la angioplastia ha mostrado resultados pobres en el tratamiento de la $\mathrm{DCE}^{(13)}$. Los riesgos de la intervención son la propagación iatrogénica del hematoma intramural a partir del traumatismo generado por la angioplastia, y, en menor medida, el riesgo de entrar en la falsa luz con la guía de angioplastia. El fracaso técnico de la angioplastia fue de 53\% en una serie de 189 pacientes ${ }^{(11)}$ y de $64 \%$ en la serie de Vancouver $^{(12)}$. La cirugía de revascularización miocárdica en agudo se reserva para los casos de inestabilidad clínica con isquemia persistente en los que la angioplastia está contraindicada o sufrió alguna complicación. Una serie retrospectiva de la Clínica Mayo mostró una tasa de éxito de 94\% con una sobrevida intrahospitalaria de $100 \%{ }^{(11)}$. A la luz del conocimiento fisiopatológico actual, no sorprende que en el seguimiento los pacientes operados muestren una baja tasa de permeabilidad de los bypass, probablemente debido a la curación espontánea de la disección y el restablecimiento del flujo sanguíneo coronario normal a través del lecho nativo(10).

Durante el tiempo de seguimiento, no se registraron recurrencias o eventos cardiovasculares adversos, permaneciendo vivos y asintomáticos todos los pacientes que sobrevivieron al evento inicial. La recurrencia de DCE muestra diferencias en las distintas series y va desde 5\% a dos años hasta $30 \%$ cuando el seguimientos es de cuatro a diez años ${ }^{(13)}$.

\section{Limitaciones del estudio}

Se trata de un estudio realizado en un solo centro, con un número relativamente bajo de casos identifi- 

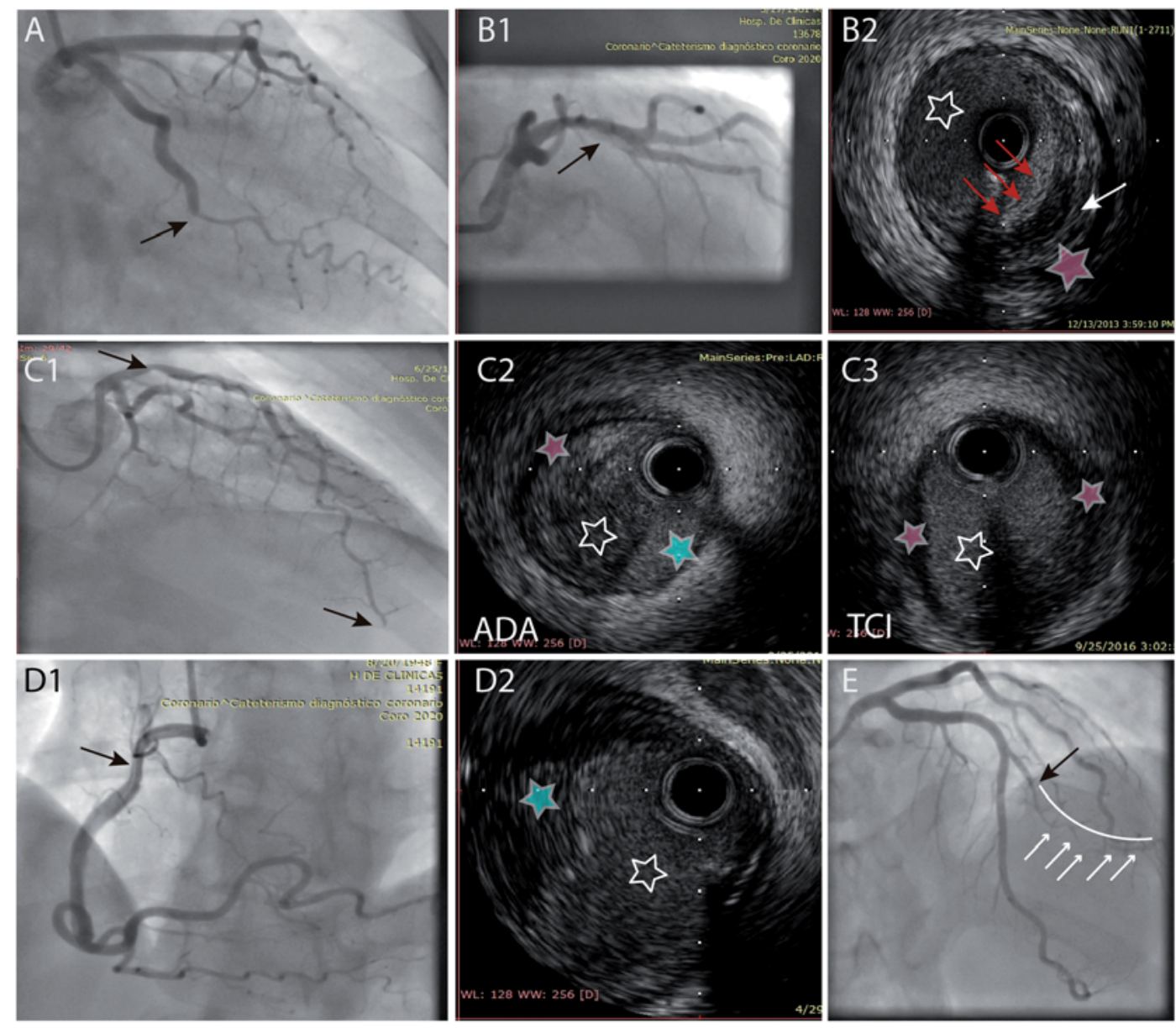

Figura 3. A) Imagen angiográfica de disección tipo 2b. Se observa a nivel de la flecha negra el sitio de inicio de la DCE, hay un afinamiento del vaso, incremento de su tortuosidad y no recupera calibre. B1) Imagen angiográfica de disección tipo 3. Lesión focal con aspecto de trombo que afecta la bifurcación de arteria descendente anterior con primer ramo diagonal. B2) IVUS muestra hematoma intramural señalado con estrella rosa, complejo intimomedial señalado con flecha blanca y por dentro imagen compatible con trombo que estenosa parcialmente la luz. La luz verdadera se señala mediante la estrella blanca. Correspondería a disección tipo 3. C1) Imagen angiográfica de estenosis focal, señalada con la flecha negra superior, y detención abrupta de flujo a nivel apical en la arteria descendente anterior, flecha negra inferior. C2) IVUS muestra a nivel de ADA proximal la existencia de un hematoma intramural (estrella rosada), sector correspondiente a trombo que estenosa parcialmente la luz (estrella celeste) y la luz verdadera (estrella blanca). C3) IVUS muestra a nivel de TCI el origen del hematoma intramural a dicho nivel, que se observa como un espacio ecolúcido ancho en la pared arterial (estrellas rosadas), corresponde a una disección tipo 3 con oclusión distal. D1) Se observa la típica imagen angiográfica de doble luz, correspondiente a una disección tipo 1. D2) Muestra la presencia de una luz verdadera (estrella blanca) y una luz falsa (estrella celeste). E) Imagen angiográfica compatible con disección tipo 2a. Existe un estrechamiento abrupto del primer ramo diagonal (flecha negra), que en su sector medio se hace filiforme y posteriormente recupera calibre (flechas blancas). La línea blanca representa el trayecto del primer ramo diagonal.

cados que impide realizar una descripción generalizable. El carácter retrospectivo de búsqueda a través de bases de datos impide identificar casos no reconocidos por el hemodinamista actuante, por lo que la incidencia real es probablemente más alta.

\section{Conclusión}

Se trata del primer reporte en nuestro país sobre DCE, analizando, en un solo centro, su incidencia, características clínicas, angiográficas, imagenológicas, tratamiento y evolución.
Se demostró una baja incidencia de DCE como causa de SCA; sin embargo, se debe tener presente como diagnóstico diferencial, principalmente en mujeres de mediana edad. La importancia de este diagnóstico radica en que el tratamiento y la evolución difieren de los de la complicación de la enfermedad aterosclerótica. 
Contribución de autores

Rafael Mila, https://orcid.org/0000-0003-1607-471X

Idea original y diseño del trabajo, recolección de datos, revisión y creación de la base de datos, interpretación de resultados, redacción del manuscrito.

Juan Albistur, https://orcid.org/0000-0002-0282-5263

Recolección de datos, revisión y creación de la base de

datos, interpretación de resultados y aprobación del manuscrito final.

Juan Pablo Bachini,

https://orcid.org/0000-0001-7278-8691 Recolección de datos, revisión y creación de la base de datos, interpretación de resultados y aprobación del manuscrito final. Álvaro Niggemeyer, https://orcid.org/0000-0003-0911-6029 Aprobación del manuscrito final.

Ariel Durán, https://orcid.org/0000-0003-1293-2000

Supervisión general del trabajo y aprobación del manuscrito final.

\section{Bibliografía}

1. Saw J, Mancini G, Humphries K. Contemporary review on spontaneous coronary artery dissection. J Am Coll Cardiol. 2016; 68(3):297-312. doi: 10.1016/ j.jacc.2016.05.034

2. Saw J, Aymong E, Mancini G, Sedlak T, Starovoytov A, Ricci D. Nonatherosclerotic coronary artery disease in young women. Can J Cardiol. 2014; 30(7):814-9. doi: 10.1016/j.cjca.2014.01.011

3. Saw J. Spontaneous coronary artery dissection. Can J Cardiol. 2013; 29(9):1027-33. doi: 10.1016/j.cjca. 2012.12.018

4. Cerrato E, Giacobbe F, Rolfo C, Quadri G, Tomassini F, Ferrari F, et al. Role of Invasive and Non-invasive Imaging Tools in the Diagnosis and Optimal Treatment of Patients with Spontaneous Coronary Artery Dissection. Curr Cardiol Rep. 2019; 21(10):122. doi: 10.1007/s11886-019-1202-0

5. Al-Hussaini A, Adlam D. Spontaneous coronary artery dissection. Heart 2017; 103(13):1043-51. doi: 10.1136/heartjnl-2016-310320

6. Alfonso F, Dutary J, Paulo M, Gonzalo N, Pérez-Vizcayno $M$, Jiménez-Quevedo $P$, et al.
Combined use of optical coherence tomography and intravascular ultrasound imaging in patients undergoing coronary interventions for stent thrombosis. Heart 2012; 98(16):1213-20. doi: 10.1136/heartjnl2012-302183

7. Alfonso F, Paulo M, Lennie V, Dutary J, Bernardo E, Jiménez-Quevedo P, et al. Spontaneous coronary artery dissection: long-term follow-up of a large series of patients prospectively managed with a "conservative" therapeutic strategy. JACC Cardiovasc Interv. 2012; 5(10):1062-70. doi: 10.1016/j.jcin. 2012.06.014

8. Saw J, Starovoytov A, Humphries K, Sheth T, So D, Minhas K, et al. Canadian spontaneous coronary artery dissection cohort study: in-hospital and 30-day outcomes. Eur Heart J. 2019; 40(15):1188-97. doi: 10.1093/eurheartj/ehz007

9. Waterbury T, Tarantini G, Vogel B, Mehran R, Gersh B, Gulati R. Non-atherosclerotic causes of acute coronary syndromes. Nat Rev Cardiol. 2019; 3:10. 1038/s41569-019-0273-3. doi: 10.1038/s41569-0190273-3

10. Franke K, Wong D, Baumann A, Nicholls S, Gulati R, Psaltis P. Current state-of-play in spontaneous coronary artery dissection. Cardiovasc Diagn Ther. 2019; 9(3):281-98. doi: 10.21037/cdt.2019. 04.03.

11. Tweet M, Hayes S, Pitta S, Simari R, Lerman A, Lennon R, et al. Clinical features, management, and prognosis of spontaneous coronary artery dissection. Circulation 2012; 126(5):579-88. doi: 10.1161/ CIRCULATIONAHA.112.105718

12. Saw J, Aymong E, Sedlak T, Buller C, Starovoytov A, Ricci D, et al. Spontaneous coronary artery dissection: association with predisposing arteriopathies and precipitating stressors and cardiovascular outcomes. Circ Cardiovasc Interv. 2014; 7(5):645-55. doi: 10.1161/CIRCINTERVENTIONS.114.001760

13. Hayes S, Kim E, Saw J, Adlam D, Arslanian-Engoren $\mathbf{C}$, Economy $\mathbf{K}$, et al. Spontaneous coronary artery dissection: current state of the science: a scientific statement from the American Heart Association. Circulation 2018; 137(19):e523-57. doi: 10.1161/CIR.0000000000000564 